Notfall Rettungsmed 2009 · 12:336-338

DOI 10.1007/s10049-009-1219-9

Online publiziert: 23. Juli 2009

๑) Springer Medizin Verlag 2009

\author{
U. Kreimeier ${ }^{1}$ B B. Dirks ${ }^{2}$ \\ ${ }^{1}$ Klinik für Anästhesiologie, Klinikum der Universität München1 \\ 2 Sektion Notfallmedizin, Klinik für Anaesthesiologie, Universitätsklinikum
}

\title{
Lebenslanges Lernen - ein Konzept für die Notfallmedizin
}

\begin{abstract}
Lebenslanges Lernen hat in den letzten Jahren zunehmende Aufmerksamkeit auf sich gezogen und Einzug in viele bildungspolitische Forderungen und Konzepte gehalten. „Lifelong learning“ (LLL) ist das lebenslange, eigenmotivierte Lernen in allen Lebensbereichen. Lebenslanges Lernen sollte wirkungsvoll und nachhaltig sein. Übertragen auf die Medizin bedeutet das Konzept des „lifelong learning“, dass die Ausbildung im Gesundheitssystem nicht mit Erreichen des Berufsziels (Facharzt, Rettungsassistent u. a.) abgeschlossen ist, sondern sich in einem kontinuierlichen Prozess der Aktualisierung des Fachwissens befinden muss, der mit dem (Eigen-)Anspruch zum Erlernen auch neuer Techniken verbunden sein sollte.
\end{abstract}

\section{Qualität in der Notfallmedizin}

Die Versorgungsqualität in der Notfallmedizin wird meist an numerischen Einsatz- und Versorgungsdaten (Alarmierungszeit, präklinische Versorgungszeit, „door to needle“-time u. a.) gemessen. Schnelligkeit wird hierbei mit Güte der Versorgung assoziiert. Bezeichnend ist ein Motto wie „time is brain“ im Falle des Schlaganfalls - je länger die Zeitspanne bis zur definitiven Therapie (Desobliteration) ist, umso ungünstiger ist schlussendlich die Prognose, im Falle des Apoplex das neurologische Outcome und damit die Lebensqualität des Patienten im Alltag. Schnelligkeit setzt Sicherheit der Maßnahmen voraus. Sicherheit entsteht langfristig durch Erfahrung, kann aber zu Beginn nur durch intensives Training erworben werden. Präklinische wie klinische notfallmedizinische Versorgung betreffen allerdings auch seltene lebensbedrohende Situationen, auf die der Notfallmediziner nicht aus der Routine vorbereitet ist und die somit dauerndes intensives Weiterlernen nach Abschluss der Ausbildung erfordern.

Notfallmedizin ist als Teamwork und als Einheit aus präklinischer und früher klinischer Notfallversorgung zu sehen. Therapeutische Maßnahmen werden damit im Team und teamübergreifend durchgeführt und erfordern eine durchgängige Kommunikation, um einen effizienten Ablauf auch an den Schnittstellen (Übergabe an ein anderes Behandlungsteam) sicherzustellen.

\section{"Continuous education" in der Notfallmedizin}

Andre Gries et al. [3] haben 2005 unter Berücksichtigung einer Befragung während der INTECH-Fortbildung sowie basierend auf einer großen Zahl von Einsatzdaten einen Überblick veröffentlicht, wie häufig Notärzte mit bestimmten kritischen Einsatzsituationen und den dafür notwendigen Maßnahmen im Notarztdienst und der Luftrettung konfrontiert sind. Das Ergebnis lässt einen hohen Bedarf für lebenslanges Lernen erkennen.

Einige Eckpunkte: Während das akute Koronarsyndrom und der Apoplex dem Notarzt im Durchschnitt 2-mal pro Monat begegnen, muss der Bodennotarzt nur 1-mal pro Jahr ein Polytrauma und nur 7- bis 8-mal pro Jahr einen Kindernotfall oder eine Reanimation meistern. Eine Thoraxdrainage legt der Bodennotarzt statistisch alle 8o Monate! Dieses Routinedefizit kann nur durch intensive Fortund Weiterbildung ausgeglichen werden, bis zum Ende der Tätigkeit, lebenslang.

\section{Ohne regelmäßige Weiterbildung wird das Risiko für den Patienten unkalkulierbar}

Andernfalls wird das Risiko, das wir dem Patienten zumuten, unkalkulierbar, die Güte der Versorgung sicher schlecht. INTECH ist der Typ von Veranstaltung, die diesem erkannten Mangel entgegensteuern mit dem Schwerpunkt auf praktischer Fortbildung. Es ist alles andere als trivial, wie man mit vertretbarem Aufwand einen guten Fortbildungseffekt erzielt - einerseits wegen der Notwendigkeit praktischer Erfahrung, andererseits wegen der spezifischen Probleme der Erwachsenenbildung und der kurzen Halbwertszeit des Erlernten und Geübten.

\section{Lebenslanges Lernen am Beispiel von Basic Life Support}

Auch in der Laienhilfe ist die Vermittlung von Kenntnissen in Erster Hilfe nicht gelöst. Wir haben in Deutschland die gute Gelegenheit, jedem jungen Erwachsenen anlässlich seines Führerscheinerwerbs die Maßnahmen der Ersten Hilfe vermit- 
teln zu können. Was bleibt hängen und wie lange? Jan Breckwoldt et al. $[1,2]$ sind in Berlin dieser Frage im Rahmen einer Beobachtungsstudie von Laienhelfern in Reanimationssituationen nachgegangen. Die Ergebnisse sind nicht ermutigend. Sie werden in Kürze mehr darüber erfahren. Aus den Beobachtungen muss man den Schluss ziehen, dass wir die Lehrmethoden und Schwerpunkte der Ausbildung überdenken müssen.

\section{Crew Resource Management}

Die Luftfahrt und das von ihr entwickelte Cockpit bzw. Crew Resource Management (CRM) als Werkzeug für sicheres Handeln des Einzelnen, von Teams und der Luftfahrtindustrie als Ganzem werden gerne als Beispiel für ein erfolgreiches Konzept zur Risikosteuerung verwendet. Ausgehend von der Betrachtung der Zusammenarbeit im Cockpit eines Flugzeuges (Cockpit Resource Management) wurden die Schnittstellen zum Cockpit einbezogen (Crew Resource Management) und das Training letztendlich auf alle Arbeitsbereiche, die zum System Luftfahrt gehören, ausgedehnt.

Die Konzepte Cockpit/Crew Resource Management (CRM) und Multi Crew Coordination (MCC) haben seit langem Einzug in die moderne Fliegerei gefunden. Aus der Aufzeichnung von Zwischenfallsimulation im Cockpit großer amerikanischer Militärmaschinen ist bekannt, dass die Kommunikation zwischen den Crew-Mitgliedern ein wichtiges Element zur Bewältigung von Aufgaben ist, und Teamtraining zu einer Steigerung der Anzahl abgearbeiteter Einzelaufgaben und zu einer besseren Bewältigung von komplexen Situationen führt. Festgelegte Check-Abfolgen, festgelegte verbale und nonverbale Crew-Kommunikation etc. gehören zum Standard der Aus- und Weiterbildung von Jet-Piloten.

Komplexe Situationen werden auch bei der Behandlung von Notfallpatienten immer häufiger. Die klinische Versorgung von Notfallpatienten ist durch neue diagnostische und therapeutische Techniken einer stetigen Weiterentwicklung und Modernisierung unterworfen, um selbst organübergreifende, generalisierte Krankheitsbilder schnell erkennen und effektiv behandeln zu können. Hierzu sind nicht selten komplexe Handlungsabläufe not- wendig, die präklinisch wie klinisch auch hohe Anforderungen an die Kommunikation des Notfallteams stellen.

\section{"Team leadership" und Koordination der Einzelmaßnahmen}

Bekanntes Beispiel für eine hierarchische Struktur ist das etablierte Konzept der Polytraumaversorgung, die durch einen „team leader“koordiniert wird. Der „team leader" trägt die Verantwortung für die Gesamtkoordination und Einleitung der nächsten Behandlungsschritte. Ein wichtiges Element in Übungsszenarien bei modernen Lernmodellen ist die Auflösung der hierarchischen Strukturen und die Hinführung zur Zuteilung von Prozessabläufen, die von einzelnen Teams eigenverantwortlich bearbeitet und dann rückgemeldet werden. Durch eine klare und eindeutige verbale und nonverbale Kommunikation erfolgt ein Feedback an einen koordinierenden "supervisor“, der die Daten aus Vitalparametern, Diagnostik sowie von Personal- und Raumressourcen abgleicht und die nächsten Behandlungsschritte vorgibt. Der „team leader“ entwickelt sich hin zum Koordinator der notfallmedizinischen Maßnahmen, der zukünftig ein Team an seiner Seite hat, das aus der praktischen Erfahrung zusammen mit dem Training von Standardsituationen heraus Entscheidungsabläufe selbstständig evaluiert und einen bestmöglichen Weg findet.

Während derzeit noch das Patientenmanagement fachrichtungsspezifisch gegliedert ist, werden aktuell Teamaufgaben diskutiert, die fachrichtungsübergreifend problemorientiert gelöst werden. So wird bei der Polytraumaversorgung diskutiert, feste Behandlungsteams für Atemweg, Kreislauf und Bildgebung einzusetzen. Hier ist zunehmend interdisziplinäres Denken und Handeln gefragt, das nachhaltig geübt werden muss.

\section{(7) Für das gesamte Notfallteam ist ein kontinuierlicher Lernprozess erforderlich}

Strukturen und Prozesse in der Notfallmedizin sind - nicht zuletzt aufgrund der rasanten technischen Entwicklungen

\section{Infobox 1}

\section{Resuscitation 2009}

„Education“ ist das zentrale Thema des diesjährigen gemeinsamen Kongresses Resuscitation 2009 des European Resuscitation Council (ERC) und des German Resuscitation Council (GRC), der vom 2.-3. Oktober in Köln stattfindet. Internationale Experten werden Ihnen außerdem einen ersten Ausblick auf die International Liaison Committee on Resuscitation (ILCOR) -Leitlinienkonferenz im Februar 2010 in Dallas geben. Konzepte und Ergebnisse aus dem Gebiet Unterricht, Ausbildung und Training in der Notfallmedizin werden behandelt. Die aktuellen Planungen für die ERC-Ausbildungskonzepte und neue Kurse wie Immediate Life Support (ILS), European Pediatric Immediate Life Support (EPILS) oder Prehospital European Trauma Course (pETC) werden vorgestellt.

Weitere Informationen:

www.resuscitation2009.eu

- einer ständigen Erweiterung und Überarbeitung unterworfen. Dazu ist ein kontinuierlicher Lernprozess für das gesamte Notfallteam erforderlich. Das erworbene Wissen muss möglichst realitätsnah geübt werden. MitHilfe von Full-Scale-Patientensimulatoren werden am Modell (patho)physiologische Reaktionen dargestellt, so dass nicht nur Grundtechniken wie Reanimation oder Intubation, sondern auch Teamwork trainiert werden können. Es sollte nicht unerwähnt bleiben, dass Simulatoren teuer sind. Sie haben sich aber als absolut kosteneffizient herausgestellt - ein Ergebnis wiederum aus dem Bereich der Luftfahrt!

\section{Bedeutung der "soft skills"}

Ärzte aller Fachrichtungen sind regelmäBig mit Belastungssituationen und potenziell mit Zwischenfällen konfrontiert. Die Kompetenz der Behandler muss nicht nur medizinisch-fachliches Wissen, sondern auch Problemlösefähigkeiten, Teamführungsfähigkeiten und andere "soft skills“ umfassen. Von essentieller Bedeutung beim CRM ist das praxisnahe, authentische Training von Krisensituationen im Team. Durch gezieltes Training von simulierten Situationen, die standardisiert herbeigeführt werden, ist eine effiziente Ausbildung mit einem hervorragenden Lerneffekt gegeben. Derartige Trainingszenarien haben das Üben am Patienten, das dem 
Erlernen standardisierter Handgriffe und Handlungsabläufe dient, in vielen Bereichen abgelöst.

Teamassoziiertes Training notfallmedizinischer Standardsituationen dient somit:

- der Sicherung der Versorgungsqualität,

— der Erhöhung der Patientensicherheit und

- der Förderung der Mitarbeitermotivation.

\section{Notfallmedizin „goes" lebenslanges Lernen!}

Nicht allein der Arbeitsplatz des Anästhesisten, des Kardiologen, Radiologen und vieler anderer Fachgebiete ist mehr und mehr technisiert und ähnelt in weiten Bereichen dem Cockpit von Flugzeugen. Ähnliches gilt für die Monitoring- und Behandlungsoptionen im modernen Rettungswagen, Notarzteinsatzwagen, Intensivtransportwagen oder Rettungshubschrauber/Intensivtransporthubschrauber. Das Notfallteam muss sich diesen Neuerungen stellen und sich damit vertraut machen. Die zentrale Bedeutung von Unterricht, Ausbildung und kontinuierlichem Training ist mittlerweile auch in der Notfallmedizin unbestritten, da hierdurch die Prozessqualität in der Versorgung der Patienten ganz wesentlich bestimmt wird. Lebenslanges notfallmedizinisches Lernen eben!

\section{Supplement „Education“ von Notfall + Rettungsmedizin}

Unterricht, Ausbildung und Training sind darüber hinaus wichtige Elemente im Qualitätsmanagement und in der Qualitätssicherung bei der Behandlung von Notfallpatienten. Der German Resuscitation Council (GRC), dessen offizielles Organ unsere Zeitschrift ist, gibt zusammen mit dem European Resuscitation Council (ERC) nunmehr ein erstes, englischsprachiges Supplement mit dem Titel „Education“ heraus. Dieses Supplement erscheint anlässlich des wissenschaftlichen Symposiums „Resuscitation 2009“(• Infobox 1) von ERC und GRC, das vom 2.-3. Oktober 2009 in Köln stattfindet. Hier werden aktuelle Themen aus dem Bereich Lehren und Lernen in Form von State-of-the-ArtReviews behandelt. Das Supplement wird den Abonnenten von Notfall + Rettungsmedizin Anfang Oktober automatisch und kostenlos zugehen. Neben der Bedeutung, die wir diesem Themenbereich beimessen, soll es die Führungsrolle unserer Zeitschrift im deutschsprachigen Raum in der Notfallmedizin auf wissenschaftlichem Gebiet unterstreichen und Sie, liebe Leserinnen und Leser, beim lebenslangen Lernen unterstützen!

Ihre

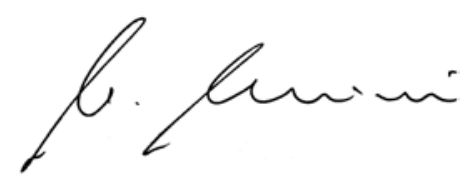

U. Kreimeier

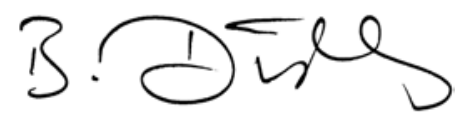

B. Dirks

\section{Korrespondenzadresse}

\section{Prof. Dr. U. Kreimeier}

Klinik für Anästhesiologie, Klinikum der Universität München

Nussbaumstr. 20, 80336 München

uwe.kreimeier@med.uni-muenchen.de

\section{Literatur}

1. Breckwoldt J, Schloesser S. (2008) Perceptions of lay bystanders of out-of-hospital cardiac arrest (OOHCA). Resuscitation 77: 19-20

2. Breckwoldt J, Schloesser S, Müller D, Arntz H.-R. (2008) Actions of bystanders of out-of-hospital cardiac arrest (OOHCA). Resuscitation 77:7

3. Gries A, Zink W, Bernhard M, Messelken M, Schlechtriemen T. (2005) Einsatzrealität im Notarztdienst. Notfall Rettungsmed 8:391-398
RESUSCITATION

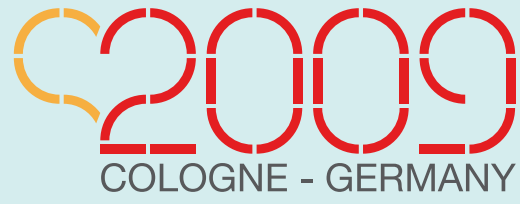

\section{Kongressankündigung}

Resuscitation 2009, 2.-3.10.2009 in Köln

Am 2. und 3. Oktober 2009 findet in Köln der internationale Kongress „Resuscitation 2009" des European Resuscitation Council (ERC) statt, vom neu gegründeten German Resuscitation Council (GRC) erstmals in Deutschland ausgerichtet.

Das zentrale Thema dieses Kongresses ist „Education". Behandelt werden aktuelle Konzepte und neue Ergebnisse aus dem Gebiet Unterricht, Ausbildung und Training in der Notfallmedizin.

Internationale Experten geben einen ersten Ausblick auf die mit Spannung erwartete International Liaison Committee on Resuscitation (ILCOR) -Leitlinienkonferenz im Februar 2010 in Dallas. Planungen für die ERC-Ausbildungskonzepte und neue Kurse wie Immediate Life Support (ILS), European Pediatric Immediate Life Support (EPILS) oder Prehospital European Trauma Course (pETC) werden ebenfalls vorgestellt.

\section{GRC-Symposium am Freitag}

Den Auftakt am Vormittag des Freitag, 2. Oktober gestaltet der GRC mit deutschsprachigen KeyLectures zu den Themen:

- ILCOR,

- rechtliche Aspekte und Ethik der Reanimation und - CPR-Techniken und Geräte.

Zum Kongress wird der GRC zusammen mit dem ERC ein englischsprachiges Supplement zum Thema „Education" der Zeitschrift „Notfall + Rettungsmedizin" herausgeben.

Informationen, Programm und Registrierung: www.resuscitation2009.eu 